Article

\title{
Fucoxanthin from Undaria pinnatifida: Photostability and Coextractive Effects
}

\author{
Anna Piovan ${ }^{1}{ }^{1}$, Roberta Seraglia $^{2}$, Bruno Bresin $^{3}$, Rosy Caniato $^{1}$ and Raffaella Filippini ${ }^{1}$ \\ 1 Department of Pharmaceutical and Pharmacological Sciences, University of Padova, \\ Via Marzolo 5, Padova 35131, Italy; E-Mails: rosy.caniato@unipd.it (R.C.); \\ raffaella.filippini@unipd.it (R.F.) \\ 2 CNR-ISTM, Corso Stati Uniti 4, Padova 35100, Italy; E-Mail: roberta.seraglia@adr.pd.cnr.it \\ 3 ARPA-FVG Regional Agency for Environmental of Friuli Venezia Giulia Region, \\ Via delle Acque 28, Pordenone 33170, Italy; E-Mail: bdbres@alice.it \\ * Author to whom correspondence should be addressed; E-Mail: anna.piovan@unipd.it; \\ Tel.: +39-049-827-6266; Fax: +39-049-827-6260.
}

Received: 11 April 2013; in revised form: 17 May 2013 / Accepted: 24 May 2013 /

Published: 29 May 2013

\begin{abstract}
Fucoxanthin is one of the most abundant carotenoids and possesses a number of beneficial medicinal qualities which include its anti-oxidant, anti-obesity and anti-cancer properties. In this study, the photostability of fucoxanthin in extracts with different chemical profiles was studied. The extracts were obtained from Undaria pinnatifida, a seaweed rich in this carotenoid, using conventional liquid solvent extraction procedures and the QuEChERS method. All the extracts contained all-trans-fucoxanthin as the major compound. Conventional procedures produced a fucoxanthin purity of lower than $50 \%$, whereas after liquid-liquid partition, PSA cleanup, and PSA and GCB cleanup (QuEChERS method) fucoxanthin purity increased to $70 \%, 86 \%$, and $94 \%$, respectively. Although in the acetone extract the initial content of fucoxanthin was the highest, results demonstrate that coextractives play an important role in enhancing the rate of photodegradation. After light exposure, the conventional extracts lost around $90 \%$ of the initial fucoxanthin content. On the other hand, the extracts obtained by the QuEChERS method showed significantly higher light stability than the conventional extracts. These results suggest that the QuEChERS method could be used and further improved to obtain more purified and stable extracts for fucoxanthin from $U$. pinnatifida.
\end{abstract}


Keywords: fucoxanthin; Undaria; photostability; coextractives; QuEChERS

\section{Introduction}

Fucoxanthin is one of the most abundant carotenoids, and contributes to more than $10 \%$ of the estimated total production of carotenoids in Nature, especially in the marine environment [1]. Fucoxanthin is a pigment, along with chlorophylls and $\beta$-carotene, widely distributed in brown algae and diatoms [2]. It has an unusual structure with an allenic bond and 5,6-monoepoxide in its molecule (Figure 1).

Figure 1. Fucoxanthin.

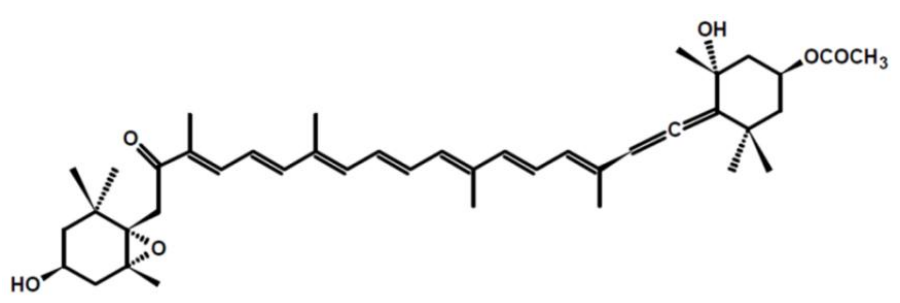

Undaria pinnatifida, a brown seaweed better known as wakame, is a rich source of fucoxanthin [3]. Actually, U. pinnatifida is widely used as a human food in many countries especially Korea and Japan, and it is becoming increasingly popular in the European market, above all in the form of extracts used as food supplements. The numerous activities attributed to $U$. pinnatifida products are essentially linked to their fucoxanthin content [4]. There is indeed a growing evidence from in vitro and in vivo experiments suggesting that fucoxanthin has health promoting effects because of its strong anti-oxidant properties [5-8]. Fucoxanthin provides protective effects on liver, blood vessels of the brain, bones, skin and eyes. It has anti-obesity and anti-diabetic properties, and anti-inflammatory and anti-malarian effects. Moreover it is very effective in inhibiting cell growth and inducing apoptosis in human cancer cells. Particularly, the anti-adult T-cell leukemia effects, the induction of apoptosis in human leukemia cells, and the anti-obesitive effect of fucoxanthin are distinctly more potent than that of $\beta$-carotene and astaxanthin. The unique effects of fucoxanthin is due its characteristic chemical structure, and carotenoids without an allenic bond are not active $[4,9,10]$. Recently, it has also been demonstrated that pretreatment with fucoxanthin improves the chemotherapeutic efficacy of cisplatin by enhancing the inhibition of cell proliferation of human hepatoma HepG2. These results suggest that the combined treatment of fucoxanthin and cisplatin may provide a novel therapeutic approach to decrease cisplatin-induced drug resistance $[11,12]$.

Owing to these properties fucoxanthin has attracted considerable interest both as nutraceuticals and pharmaceuticals, and there is an increasing interest in the effects of this seaweed carotenoid as a functional supplement in human diets helping to enhance the nutritional profiles of foods such as pasta, beverages, cakes and spreads [13-16].

Though chemical synthesis of fucoxanthin is possible, it is very expensive and therefore the viability of obtaining directly from brown seaweeds should not be overlooked [4]. Indeed there have 
been several studies which have focused on its extraction and purification from seaweeds. A crude oil, a mixture of carotenoids and polyphenols, was obtained from $U$. pinnatifida using supercritical carbon dioxide [17] and a method for its separation and purification from edible brown algae by microwave-assisted extraction coupled with high-speed countercurrent chromatography has been developed [18]. However, the most common way for fucoxanthin extraction is by liquid solvent extraction whereas the available commercial products are mainly constituted of extracts [19].

Fucoxanthin is highly susceptible to degradation and this can lead to cis-trans isomerisation, oxidative cleavage and/or epoxidation of the backbone [20]. While the deleterious effects of heat, light, or coextractives on fucoxanthin have regularly been cited for almost half a century, the extent of the impact of these factors has rarely been determined [21-23]. In this work, we have studied the effects of coextractives present in different extracts on the photostability of the fucoxanthin.

\section{Results and Discussion}

Frequent references are made in literature concerning procedures that impact on fucoxanthin stability [24-26]; however, the extent of these effects have not as yet been quantified. In this study, the photostability of the fucoxanthin in Undaria pinnatifida extracts with different chemical profiles was studied.

We used fresh starting material and not exhaustive extraction procedures in order to prevent as far as possible any degradation event occurring during the sample processing steps. The extractions were performed by using three different solvents (methanol, acetone, acetonitrile) with different extraction power both versus fucoxanthin and other compounds present in $U$. pinnatifida. The extracts were obtained in an ultrasonic bath (conventional procedures) and with the Quick, Easy, Cheap, Effective, Rugged, and Safe (QuEChERS) method [27]. The QuEChERS method is the most commonly applied prep method for the determination of pesticide residues from a variety of fruit and vegetables, fatty food matrixes like milk and eggs, and water [28]. This method involves an extraction with acetonitrile partitioned from the aqueous matrix using anhydrous magnesium sulphate $\left(\mathrm{MgSO}_{4}\right)$ and sodium chloride $(\mathrm{NaCl})$ (acetonitrile raw extract) followed by a dSPE cleanup with $\mathrm{MgSO}_{4}$ and primary secondary amine (PSA) (cleanup) or a combination of PSA and graphitized carbon black (GCB) (additional cleanup). The use of the QuEChERS method allowed us to evaluate the fucoxanthin photodegradation of increasingly purified acetonitrile extracts.

The identification of fucoxanthin in the different extracts was confirmed with an external calibration i.e., by comparing retention time, and UV-Vis and MS/MS spectra, respectively, of the samples to those of the standard. The quantitative analyses were performed by HPLC UV-Vis. The validation data of the method are shown in Table 1.

Table 1. Regression curve data, detection limit, quantification limit and reproducibility.

\begin{tabular}{ccccc}
\hline Regression curve data & Detection limit & Quantification limit & \multicolumn{2}{c}{ Reproducibility (\% RSD) } \\
\hline $\mathrm{y}=903598 \mathrm{x}+26548$ & $(\mu \mathrm{g} / \mathrm{mL})$ & $(\mu \mathrm{g} / \mathrm{mL})$ & intra-day & inter-day \\
$\mathrm{r}^{2}=0.9999$ & 0.0112 & 0.035 & $<4$ & $<7$ \\
\hline
\end{tabular}

The use of the QuEChERS method resulted in a visible cleanup of the extracts compared to the conventional procedures showing differences in color. The HPLC chromatograms of the extracts are 
shown in Figure 2. All the extracts contained all-trans-fucoxanthin as the major compound $(\mathrm{RT}=5.5 \mathrm{~min}, \mathrm{a})$ and two other minor peaks $(\mathrm{RT}=5.5-6 \mathrm{~min}, \mathrm{~b}$ and $\mathrm{c})$ around the fucoxanthin peak, which showed similar UV-Vis spectra to fucoxanthin, as reported by Fung et al. [29]. These minor peaks were identified by MS/MS spectra as the cis-isomers of fucoxanthin. A broad and tailing peak was detected in all the conventional extracts and assigned to other coeluted matrix components; this peak was not present in the extracts obtained with the QuEChERS approach. It is worth noting that the acetonitrile raw extract (QuEChERS method) was obtained from an acetonitrile aqueous solution by salt-induced phase-separation. The different polarity of the solvent in presence of salts gave a greater matrix cleanup of the extract than with the conventional procedure with acetonitrile. The impurity content was determined as a percentage of the total area of all the peaks. The conventional procedures allowed for fucoxanthin purity lower than $50 \%$, whereas in the acetonitrile raw extract (liquid-liquid partition), after PSA cleanup and improved cleanup (QuEChERS approach) fucoxanthin purity reached $70 \%, 86 \%$, and $94 \%$ respectively.

Figure 2. HPLC chromatograms (449 nm): (A) conventional extracts (acetone-black, acetonitrile-red, methanol-blue); (B) extracts obtained using QuEChERS method (acetonitrile raw extract-blue, PSA cleanup-red, GCB additional cleanup-black).
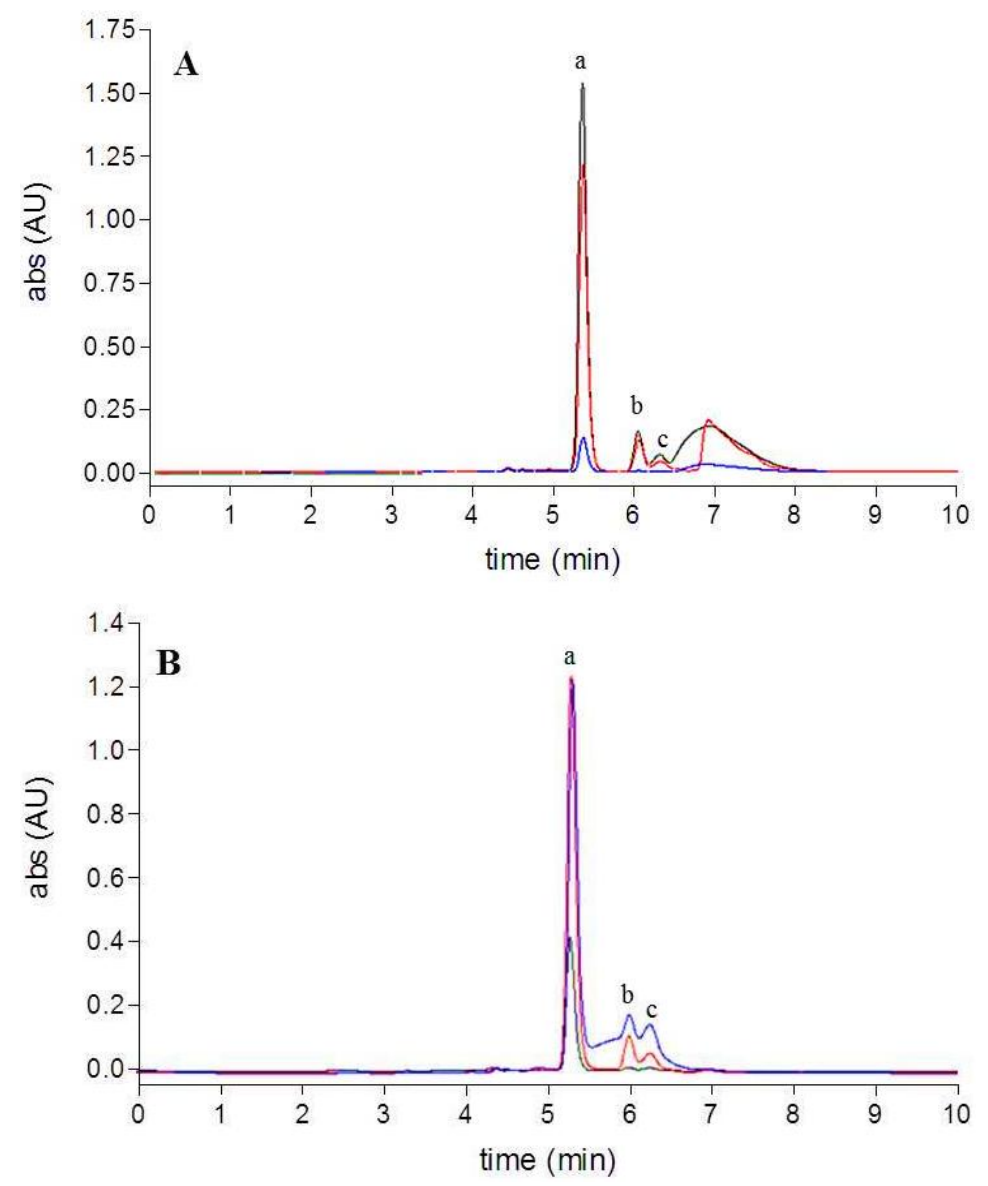

Table 2 shows the fucoxanthin contents in the different extracts. Considering the QuEChERS method, the fucoxanthin amounts in the acetonitrile raw extract and after PSA cleanup were not significantly different (using Tukey's multiple range test, $p<0.05$ ). Applying the improve cleanup with PSA and GCB, GCB had a significant effect on the recovery of fucoxanthin and drove to its 
drastic lost: only one third of the fucoxanthin was recovered with GCB compared to the acetonitrile raw extract.

Table 2. Fucoxanthin contents $(\mu \mathrm{g} / \mathrm{mL})$ in the conventional extracts (acetone, acetonitrile, methanol) and in the extracts obtained with the QuEChERS method (acetonitrile raw extract, PSA cleanup, GCB additional cleanup).

\begin{tabular}{ccccccc}
\hline \multicolumn{2}{c}{ Conventional procedures } & & \multicolumn{3}{c}{ QuEChERS method } \\
\cline { 1 - 2 } \cline { 5 - 7 } acetone & acetonitrile & methanol & & MeCN raw extract & PSA cleanup & GCB additional cleanup \\
\hline $12.3 \pm 1.10^{\mathrm{e}}$ & $9.8 \pm 0.99^{\mathrm{c}}$ & $1.0 \pm 0.12^{\mathrm{a}}$ & & $10.5 \pm 0.98^{\mathrm{c}, \mathrm{d}}$ & $10.6 \pm 1.01^{\mathrm{d}}$ & $3.3 \pm 0.29^{\mathrm{b}}$ \\
\hline
\end{tabular}

Means \pm SD. Numbers followed by the same lowercase letter did not differ statistically (Tukey test, $p>0.05$ ).

In order to evaluate the effects of coextractives on the stability of the fucoxanthin, all the extracts, fucoxanthin standard solutions and fucoxanthin standard solutions added of ascorbic acid were placed in direct daylight to compare the effects of coextractives and antioxidant ascorbic acid on fucoxanthin stability under light conditions.

Figure 3. Fucoxanthin content after light exposure expressed as a percentage of the initial content in the conventional extracts (acetone, acetonitrile, methanol; green), in the extracts obtained with the QuEChERS method (acetonitrile raw extract, PSA, GCB; grey) and in standard solutions (with and without ascorbic acid; orange).

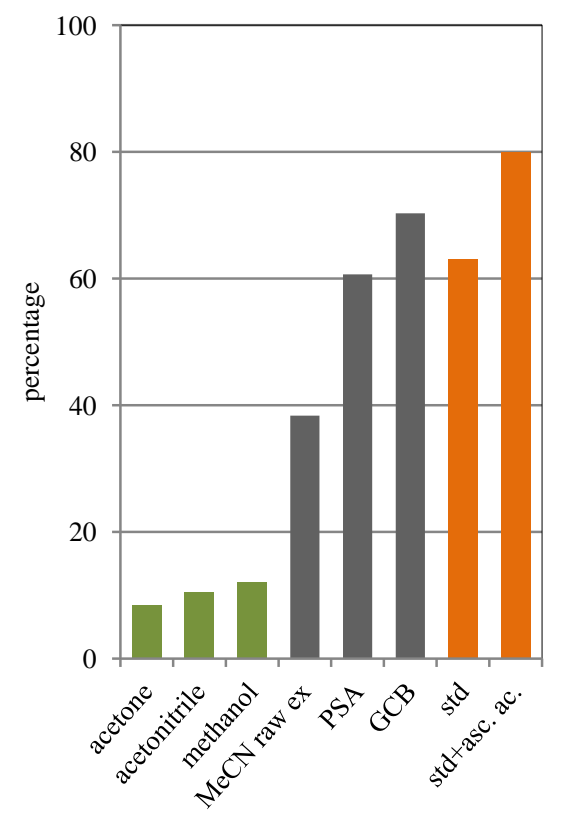

Figure 3 shows the fucoxanthin content as percentage of the initial concentration after light exposure. Results here indicate that fucoxanthin is susceptible to photodegradation. Although Mise et al. [30] reported that pure fucoxanthin is unstable but, the fucoxanthin extracted from the alga is rendered stable by the coexisting antioxidants, our data clearly demonstrate that conventional extracts are the least stable, which could be related to the matrix effects. Indeed all the solutions had lost around $90 \%$ of their initial fucoxanthin contents. In the acetonitrile raw extract (QuEChERS method) the content of fucoxanthin was reduced to $40 \%$ of its initial concentration. A steady and significant 
increase of the stability was observed after PSA cleanup and additional cleanup (PSA and GCB). The extract obtained after PSA cleanup retained around $60 \%$ of the initial concentration; in the extract obtained applying the additional cleanup the fucoxanthin was $70 \%$ of the initial concentration. The fucoxanthin standard solution and the fucoxanthin standard solution added of ascorbic acid retained around $60 \%$ and $80 \%$ of the initial concentration respectively.

As shown by the HPLC chromatograms (Figures 4 and 5), the degradation pattern of fucoxanthin in the conventional extracts on the one hand, in the extracts obtained with the QuEChERS method and in standard solutions on the other hand was notably different. Light exposure of fucoxanthin standard solutions and extracts obtained with the QuEChERS method predominantly leads to the formation of cis-isomers, whereas different unidentified compounds, displaying a retention time between 4-5 and 5.5-8 $\mathrm{min}$, are mainly formed in the conventional extracts. Again, the observed fucoxanthin loss was not mirrored by the appearance of comparable levels of isomers or degradation products as already reported [22].

Fucoxanthin is subject to isomerization and oxidation which are recognized as important reactions causing carotenoid degradation [20]. The different pattern and rate of fucoxanthin degradation occurring in the conventional extracts most probably depend on other components present in solution able to catalyze the oxidation of fucoxanthin, producing different products.

Figure 4. HPLC chromatograms $(449 \mathrm{~nm})$ obtained after light exposure of the extracts: (A) conventional extracts (acetone-black, acetonitrile-red, methanol-blue); (B) extracts obtained using QuEChERS method (acetonitrile raw extract-blue, PSA cleanup-red, GCB additional cleanup-black.
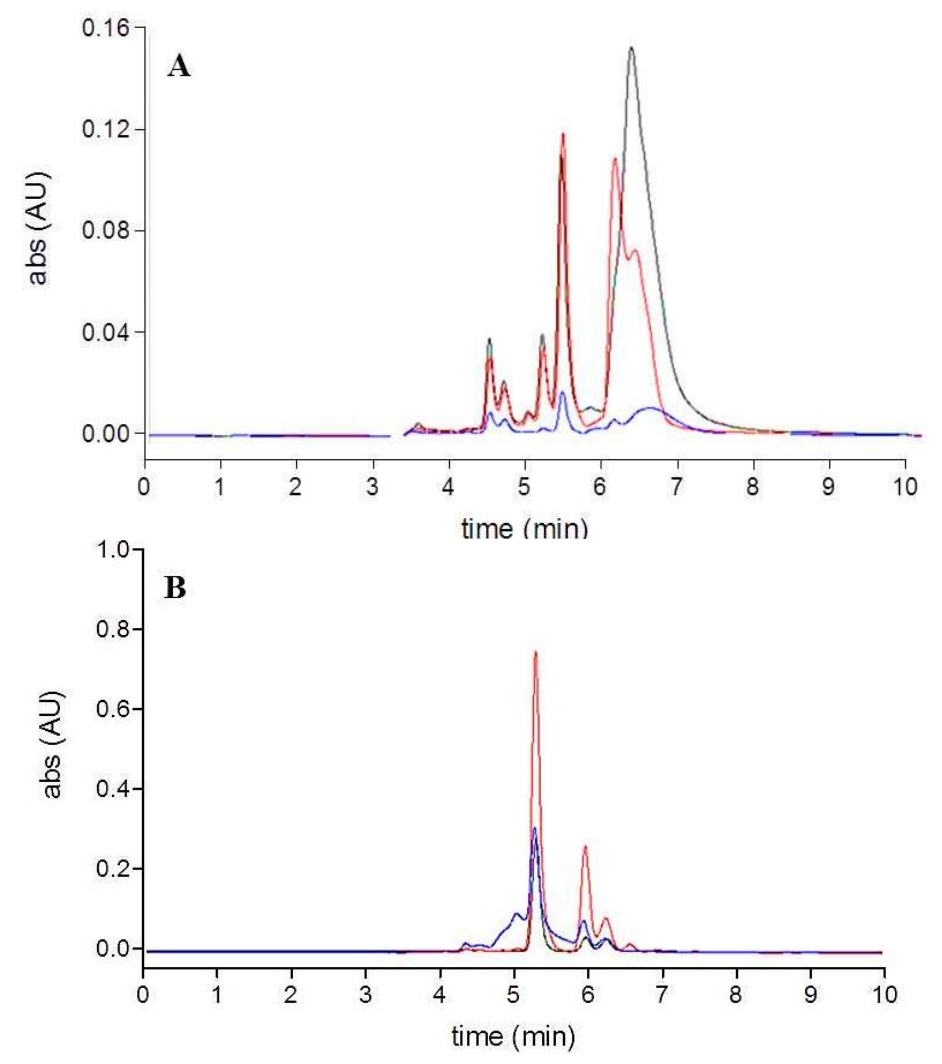
Figure 5. HPLC chromatograms $(449 \mathrm{~nm})$ of fucoxanthin standard solutions: (A) fucoxanthin standard solution-black, fucoxanthin standard solution with ascorbic acid-red; (B) after light exposure.
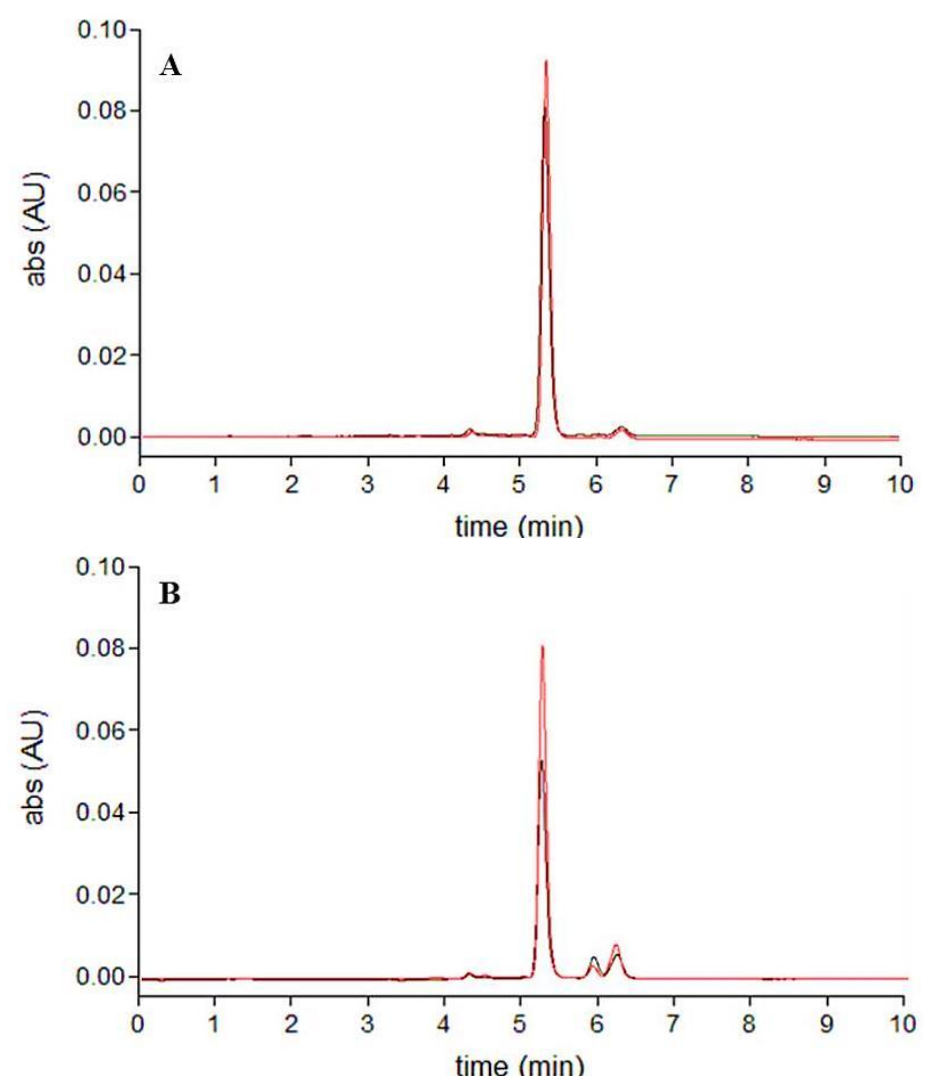

The results suggest that the first stage of degradation is the isomerization of all-trans- to more oxidable cis-isomers. Indeed in the presence of ascorbic acid, standard fucoxanthin was subject to isomerization, but the further oxidation was significantly prevented by the antioxidant agent.

On the basis of these results, a second set of experiments was carried out in order to evaluate a possible role of the water present in the conventional extracts and in the raw extract on the degradation rate. Moreover the fucoxanthin degradation rate in relation to its concentration was evaluated (Figure 6). No differences were observed in the degradation rate in pure acetonitrile solvent and in the acetonitrile/water mixture. On the contrary the results show that the degradation is faster at the higher fucoxanthin concentration. Indeed after only $30 \mathrm{~min}$ of light exposure the $10 \mu \mathrm{g} / \mathrm{mL}$ solutions had lost $20 \%$ of their initial content i.e., around $2 \mu \mathrm{g} / \mathrm{mL}$, whereas the $5 \mu \mathrm{g} / \mathrm{mL}$ solutions had lost $15 \%$ of their initial content i.e., around $0.75 \mu \mathrm{g} / \mathrm{mL}$. The contents of fucoxanthin after $5 \mathrm{~h}$ exposure were reduced by $80 \%$ and $55 \%$ respectively to around $2 \mu \mathrm{g} / \mathrm{mL}$ in all the solutions.

These results support the statement that the observed different pattern and rate of fucoxanthin degradation in the extracts depend on coextractives present in solution rather than the presence of water or fucoxanthin concentration. Indeed the extracts obtained by conventional procedures with acetone and acetonitrile, and the acetonitrile raw extract and the extract obtained by PSA cleanup (QuEChERS method) had a similar initial fucoxanthin content but underwent different rates of degradation. Moreover, the extract obtained after additional cleanup (PSA and GCB) $(3 \mu \mathrm{g} / \mathrm{mL}$ ) having a fucoxanthin purity of $94 \%$ showed a degradation rate very similar to the standard solutions $(5 \mu \mathrm{g} / \mathrm{mL})$. 
Figure 6. Fucoxanthin content after light exposure expressed as percentage of the initial content in standard solutions ( 5 and $10 \mu \mathrm{g} / \mathrm{mL})$ in pure acetonitrile solvent $(\mathrm{MeCN})$ and in acetonitrile/water mixture $(1: 1, \mathrm{v} / \mathrm{v})\left(\mathrm{MeCN}+\mathrm{H}_{2} \mathrm{O}\right)$.

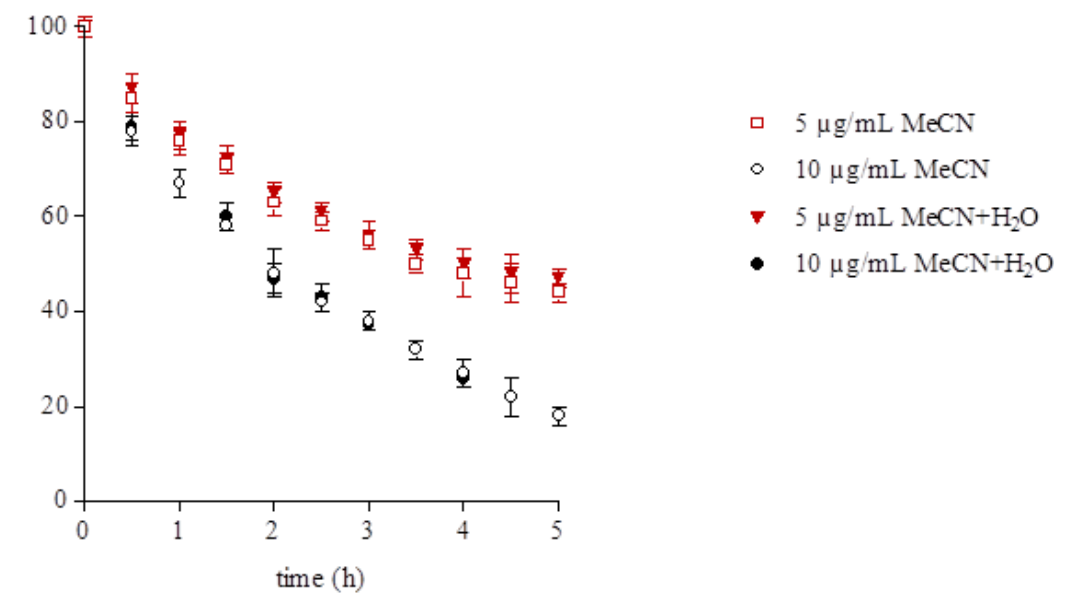

Table 3 shows the fucoxanthin content in the different extracts after light exposure. It needs to be pointed out that, although in the acetone extract (conventional procedure) the initial content of fucoxanthin was the highest $(12.3 \mu \mathrm{g} / \mathrm{mL})$, the coextractives played an important role in enhancing the rate of photodegradation. After only $90 \mathrm{~min}$ of light exposure, the content of fucoxanthin was reduced by $90 \%$ to $1.0 \mu \mathrm{g} / \mathrm{mL}$. On the other hand, even if the extract obtained after PSA cleanup (QuEChERS method) had a lower fucoxanthin content $(10.6 \mu \mathrm{g} / \mathrm{mL})$, the extract showed significantly increased light stability since the fucoxanthin content after light exposure was decreased by $40 \%$ to $6.4 \mu \mathrm{g} / \mathrm{mL}$. As pointed out above, there was no significant difference between the fucoxanthin degradation rate in the extract obtained after PSA cleanup and in standard solution, and the fucoxanthin content after light exposure was around $60 \%$ of the initial concentrations in both the samples.

Table 3. Fucoxanthin content $(\mu \mathrm{g} / \mathrm{mL})$ in the conventional extracts (acetone, acetonitrile, methanol) and in the extracts obtained with the QuEChERS method (acetonitrile raw extract, PSA cleanup, GCB additional cleanup) after light exposure.

\begin{tabular}{|c|c|c|c|c|c|}
\hline \multicolumn{3}{|c|}{ Conventional procedures } & \multicolumn{3}{|c|}{ QuEChERS method } \\
\hline acetone & acetonitrile & methanol & MeCN raw extract & PSA cleanup & GCB additional cleanup \\
\hline $1.0 \pm 0.07^{b}$ & $1.0 \pm 0.08^{b}$ & $0.1 \pm 0.01^{\mathrm{a}}$ & $4.0 \pm 0.30^{\mathrm{d}}$ & $6.4 \pm 0.57^{\mathrm{e}}$ & $2.3 \pm 0.18^{\mathrm{c}}$ \\
\hline
\end{tabular}

\section{Experimental}

\subsection{Materials}

Solvents: methanol was purchased from Merck; acetonitrile and acetone from Fluka. Water was purified with a Milli-Q deionization unit (Millipore). All-trans-fucoxanthin with $\geq 95 \%$ purity was purchased from Sigma-Aldrich.

QuEChERS materials were obtained from commercial suppliers. For the initial extraction step, QuEChERS Extraction SPE Kits (Agilent, Santa Clara, CA, USA) were used consisting of $50 \mathrm{~mL}$ 
plastic centrifuge tubes containing $4 \mathrm{~g}$ anhydrous magnesium sulphate $\left(\mathrm{MgSO}_{4}\right)$ and $1 \mathrm{~g}$ sodium chloride $(\mathrm{NaCl})$. For cleanup Q-sep ${ }^{\mathrm{TM}}$ QuEChERS dSPE Tubes for Extract Clean-Up (Restek) were used consisting of $2 \mathrm{~mL}$ mini-centrifuge tubes containing $150 \mathrm{mg}$ anhydrous $\mathrm{MgSO}_{4}, 25 \mathrm{mg}$ primary secondary amine (PSA) sorbent, with and without $7.5 \mathrm{mg}$ graphitized carbon black (GCB).

Undaria pinnatifida samples were harvested from the lagoon of Venice (Italy) in June 2012.

\subsection{Standard Stock Solution}

A stock solution of fucoxanthin was prepared in acetonitrile at a concentration of $1 \mathrm{mg} / \mathrm{mL}$ and stored at $-20{ }^{\circ} \mathrm{C}$ in amber-colored vials to protect fucoxanthin from light. The working solutions were accurately diluted with acetonitrile just prior to use.

\subsection{Sample Preparation}

The collected seaweed samples were washed with water three times. The cleaned samples were stored at $-20{ }^{\circ} \mathrm{C}$ until use. Brown seaweed samples (about $500 \mathrm{~g}$ ) made up of whole algae were homogenised with an Ultraturrax (Janke \& Kunkel IKA Labortechnik, Staufen, Germany). Extractions were performed using conventional procedures and the QuEChERS approach.

Conventional procedures: homogenised seaweed samples (10 g) were extracted with acetonitrile, methanol and acetone $(10 \mathrm{~mL})$ in an ultrasonic bath at room temperature $(10 \mathrm{~min})$.

QuEChERS approach: the original method entailed the following steps: (a) weigh $10 \mathrm{~g}$ of thoroughly homogenized sample into a $50 \mathrm{~mL}$ centrifuge tube; (b) add $10 \mathrm{~mL}$ acetonitrile; (c) add $4 \mathrm{~g}$ anhydrous $\mathrm{MgSO}_{4}$ and $1 \mathrm{~g} \mathrm{NaCl}$; shake vigorously for $1 \mathrm{~min}$ by hand; (d) centrifuge the tube at $3000 \mathrm{rpm}$ for $1 \mathrm{~min}$; (e) transfer $1 \mathrm{~mL}$ of the upper organic phase (raw extract) to a mini-centrifuge tube and subject it to a dispersive cleanup by mixing it with $150 \mathrm{mg}$ anhydrous $\mathrm{MgSO}_{4}, 25 \mathrm{mg}$ PSA; shake by hand for $30 \mathrm{~s}$; (f) centrifuge the tube at 3,000 rpm for $5 \mathrm{~min}$; transfer the final extract in a vial for analysis. A first set of extracts was prepared according to the original QuEChERS method; in a second set dSPE was performed using a combination of PSA and GCB (additional cleanup).

The extractions were performed in triplicates. The extracts were conveniently diluted before the analyses.

\subsection{Light Exposure Experiments}

In a first set of experiments, freshly prepared $1 \mathrm{~mL}$ aliquots of extracts, and standard fucoxanthin solutions $(10 \mu \mathrm{g} / \mathrm{mL})$ with and without ascorbic acid (equimolar concentration) were placed in clear glass sample vials at room temperature in direct daylight (2,500 lux; $90 \mathrm{~min})$.

In a second set of experiments standard fucoxanthin solutions ( 5 and $10 \mu \mathrm{g} / \mathrm{mL}$ ) in pure acetonitrile solvent and in acetonitrile/water mixture $(1: 1, \mathrm{v} / \mathrm{v})$ were placed in clear glass sample vials at room temperature in direct daylight (2,500 lux). Samples were collected at $30 \mathrm{~min}$ intervals for $5 \mathrm{~h}$. The experiments were performed in triplicates. 


\subsection{HPLC UV-Vis Analysis}

\subsubsection{Methodology}

HPLC analysis was performed on ChromQuest (Thermoseparation, San Jose, CA, USA) pump P4000 equipped with a photodiode array detector UV6000. The data were recorded and processed using ChromQuest Chromathography Workstation. The separation was achieved with a reversed-phase analytical column, Gemini C6-Phenyl column $(250 \times 4$, $60 \mathrm{~mm}$ i.d., $5 \mu \mathrm{m}$; Phenomenex, Torrance, CA, USA), using an isocratic elution, and the injection volume was $20 \mu \mathrm{L}$. The mobile phase was composed of methanol:water, 90:10; the flow rate was $1 \mathrm{~mL} / \mathrm{min}$. UV-Vis spectra were recorded in the 200-700 nm range; chromatograms were acquired at 449, 330 and $254 \mathrm{~nm}$.

\subsubsection{Method Validation}

The linearity of the method was evaluated by using six calibration standard solutions over a range of $0.05-7 \mu \mathrm{g} / \mathrm{mL}$. The calibration curve was established by plotting peak area ratios of calibration solutions $v s$ the nominal concentrations of fucoxanthin. The linearity was determined using linear regression analysis. The limit of detection was defined as the lowest concentration level resulting in a peak area of three times the baseline noise $(\mathrm{S} / \mathrm{N}>3)$ in solvent. The limit of quantification was defined as the lowest concentration level resulting in a peak area of ten times the baseline noise $(\mathrm{S} / \mathrm{N}>10)$ in solvent. Measurements of the intra- and inter-day variability were utilised to determine the reproducibility of the method. Fucoxanthin standard solutions were analysed to determine the intra-day repeatability (examined in one day) and inter-day repeatability (determined on 3 different days). The relative standard deviation (RSD) was calculated as a measurement of method reproducibility $(n=3)$.

\subsection{HPLC MS/MS Analysis}

The HPLC conditions were those already described. A post-column split was employed to deliver approximately $250 \mu \mathrm{L} / \mathrm{min}$ to the electrospray interface. The ESI-MS analysis was performed using a LCQ Deca Ion Trap (Thermo Finnigan, San Jose, CA, USA), operating in the positive ion mode. Sheath gas and auxiliary gas flow rates were 60 and 40 (arbitrary units) respectively, spray voltage $4 \mathrm{kV}$ and entrance capillary temperature $280{ }^{\circ} \mathrm{C}$. Collision-induced dissociation spectra were obtained applying a supplementary RF voltage, $6 \mathrm{~V}$, to the end caps of the ion trap. The data were recorded and processed using Xcalibur Workstation.

\subsection{Statistical Analysis}

Data were subjected to analysis of variance (ANOVA) to assess significant differences between the fucoxanthin content in the different extracts. Significance between the mean values was tested by Tukey's test at the confidence level of $p \leq 0.05$.

\section{Conclusions}

In this work it was demonstrated that coextractives play an important role in enhancing the rate of fucoxanthin photodegradation. After light exposure, the conventional extracts lost around 90\% of their 
initial fucoxanthin concentrations, whereas there was no significant difference between the fucoxanthin degradation rate in the extract obtained after PSA cleanup (QuEChERS method) and in standard solution with $40 \%$ loss of fucoxanthin. To the best of our knowledge, this is the first report on the application of the QuEChERS method to obtain fucoxanthin extracts from U. pinnatifida. Compared to conventional extraction techniques, the QuEChERS method provided a progressive extract cleanup and extract stability.

The promising results of fucoxanthin activity studies prompt the development of extraction and purification methods to obtain pure fucoxanthin. The above results suggest that the QuEChERS method could be used and further improved to obtain purified and stable extracts for fucoxanthin from U. pinnatifida.

\section{Acknowledgments}

This work has been supported by the MIUR. The authors are grateful to Mara Marzocchi for her valuable suggestions and Emiliano Checchin (Selc) for his assistance in harvesting Undaria pinnatifida. Proofread by Savio de Souza.

\section{Conflict of Interest}

The authors declare no conflict of interest.

\section{References}

1. Dembitsky, V.M.; Maoka, T. Allenic and cumulenic lipids. Prog. Lipid Res. 2007, 46, 328-375.

2. Takaichi, S. Carotenoids in algae: Distributions, biosyntheses and functions. Mar. Drugs 2011, 9 , 1101-1118.

3. Mori, K.; Ooi, T.; Hiraoka, M.; Oka, N.; Hamada, H.; Tamura, M.; Kusumi, T. Fucoxanthin and its metabolites in edible brown algae cultivated in deep seawater. Mar. Drugs 2004, 2, 63-72.

4. D’Orazio, N.; Gemello, E.; Gammone, M.A.; de Girolamo, M.; Ficoneri, C.; Riccioni, G. Fucoxantin: A treasure from the sea. Mar. Drugs 2012, 10, 604-616.

5. Sachindra, N.M.; Sato, E.; Maeda, H.; Hosokawa, M.; Niwano, Y.; Kohno, M.; Miyashita, K. Radical scavenging and singlet oxygen quenching activity of marine carotenoid fucoxanthin and its metabolites. J. Agric. Food Chem. 2007, 55, 8516-8522.

6. Miyashita, K.; Hosokawa, M. Beneficial Health Effects of Seaweed Carotenoid, Fucoxanthin. In Marine Nutraceuticals and Functional Foods; Shahid, F., Barrow C., Eds.; Taylor \& Francis Group: Boca Raton, FL, USA, 2008; pp. 297-308.

7. Riccioni, G. Marine carotenoids and oxidative stress. Mar. Drugs 2012, 10, 116-118.

8. Tanaka, T.; Shnimizu, M.; Moriwaki, H. Cancer chemoprevention by carotenoids. Molecules 2012, 17, 3202-3242.

9. Kim, S.K.; Pangestuti, R. Biological activities and potential health benefits of fucoxanthin derived from marine brown algae. Adv. Food Nutr. Res. 2011, 64, 111-128. 
10. Peng, J.; Yuan, J.P.; Wu, C.F.; Wang, J.H. Fucoxanthin, a marine carotenoid present in brown seaweeds and diatoms: Metabolism and bioactivities relevant to human health. Mar. Drugs 2011, 9, 1806-1828.

11. Liu, J.J.; Lu, J.; McKeage, M.J. Membrane transporters as determinants of the pharmacology of platinum anticancer drugs. Curr. Cancer Drug Targets 2012, 12, 962-986.

12. Liu, C.L.; Lim, Y.P.; Hu, M.L. Fucoxanthin enhances cisplatin-induced cytotoxicity via NFkB-mediated pathway and down regulates DNA repair gene expression in human hepatoma HepG2 cells. Mar. Drugs 2013, 11, 50-66.

13. Chandini, S.K.; Ganesan, P.; Suresh, P.V.; Bhaskar, N. Seaweeds as a source of nutritionally beneficial compounds. J. Food Sci. Technol. 2008, 45, 1-13.

14. Prabhasankar, P.; Ganesan, P.; Bhaskar, N.; Hirose, A.; Stephen, N.; Gowda, L.R.; Miyashita, K. Edible Japanese seaweed, wakame (Undaria pinnatifida) as an ingredient in pasta: Chemical, functional and structural evaluation. Food Chem. 2009, 115, 501-508.

15. Ngo, D.H.; Wijesekara, I.; Vo, T.S.; Van Ta, Q.; Kim, S.K. Marine food derived functional ingredients as potential antioxidants in the food industry: an overview. Food Res. Int. 2010, 44, 523-529.

16. Oryza. Fucoxanthin: Dietary Ingredient for Prevention of Metabolic Syndrome and Beauty Enhancement; Oryza oil \& Fat Chemical CO., LTD.: Tokyo, Japan, 2011.

17. Roh, M.K.; Uddin, S.; Chun, B.S. Extraction of fucoxanthin and polyphenol from Undaria pinnatifida using supercritical carbon dioxide with co-solvent. Biotechnol. Bioproc. Eng. 2008, 13, 724-729.

18. Xiao, X.; Si, X.; Yuan, Z.; Xu, X.; Li, G. Isolation of fucoxanthin from edible brown algae by microwave-assisted extraction coupled with high-speed countercurrent chromatography. J. Sep. Sci. 2012, 35, 2313-2317.

19. Kim, S.M.; Jung, Y.J.; Kwon, O.N.; Cha, K.H.; Um, B.H.; Chung, D.; Pan, C.H. A potential commercial source of fucoxanthin extracted from the microalga Phaeodactylum tricornutum. Appl. Biochem. Biotechnol. 2012,166, 1843-1855.

20. Davey, M.W.; Mellidou, I.; Keulemans, W. Considerations to prevent the breakdown and loss of fruit carotenoids during extraction and analysis in Musa. J. Chrom. A 2009, 1216, 5759-5762.

21. Mousseron-Canet, M.; Dalle, J.P.; Mani, J.C. Photooxydation sensibilisee de composes apparentes aux carotenoides. Analogie avec la structure des carotenoides oxygenes, alleniques et autres. Tetrahedron Lett. 1968, 9, 6037-6040.

22. Mercadante, A.Z. Carotenoids in Foods: Sources and Stability during Processing and Storage. In Food Colorants: Chemical and Functional Properties; Carmen S., Ed.; CRC Press: Boca Raton, FL, USA, 2008; pp. 213-240.

23. Rivera, S.; Canela, R. Influence of sample processing on the analysis of carotenoids in maize. Molecules 2012, 17, 11255-11268.

24. Haugan, J.A.; Liaaen-Jensen, S. Improved isolation procedure for fucoxanthin. Phytochemistry 1989, 28, 2797-2798.

25. Kim, S.J.; Kim, H.J.; Moon, J.S.; Kim, J.M.; Kang, S.G.; Jung, S.T. Characteristic and extraction of fucoxanthin pigment in Undaria pinnatifida. J. Kor. Soc. Food Sci. Nutr. 2004, 33, 847-851. 
26. Shang, Y.F.; Kim, S.M.; Lee, W.J.; Um, B.H. Pressurized liquid method for fucoxanthin extraction from Eisenia bicyclis (Kjellman) Setchell. J. Biosci. Bioeng. 2010, 111, 237-241.

27. Anastassiades, M.; Lehotay, S.J.; Stajnbaher, D.; Schenck, F.J. Fast and easy multiresidue method employing acetonitrile extraction/partitioning and "dispersive solid-phase extraction" for the determination of pesticide residues in produce. J. AOAC Int. 2003, 86, 412-431.

28. Majors, R.E. QuEChERS - A new sample preparation technique for multiresidue analysis of pesticides in foods and agricultural samples. LCGC North Am. 2007, 25, 436-446.

29. Fung, A.; Hamid, N.; Lu, J. Fucoxanthin content and antioxidant properties of Undaria pinnatifida. Food Chem. 2013, 136, 1055-1062.

30. Mise, T.; Ueda, M.; Yasumoto, T. Production of fucoxanthin-rich powder from Cladosiphon okamuranus. Adv. J. Food Sci. Technol. 2011, 3, 73-76.

Sample Availability: Samples of fucoxanthin and Undaria pinnatifida are available from the authors.

(C) 2013 by the authors; licensee MDPI, Basel, Switzerland. This article is an open access article distributed under the terms and conditions of the Creative Commons Attribution license (http://creativecommons.org/licenses/by/3.0/). 